\title{
Dépistage postnatal du diabète à la suite d'un diabète sucré gestationnel : efficacité du système de rappel
}

\author{
A. K. Shea, Ph. D., M. Sc. (1); B. R. Shah, M.D., Ph. D. (2,3,4); H. D. Clark, M. D., M. Sc. (5,7); J. Malcolm, M. D. (5); \\ M. Walker, M. D., M. Sc. (6,7); A. Karovitch, M.D., M. Ed. (5,6); E. J. Keely, M. D. (5,6)
}

\section{Résumé}

Introduction : Dans le cadre des soins de santé courants, les femmes ayant des antécédents de diabète sucré gestationnel (DSG) subissent rarement au cours de la période postnatale le test recommandé de dépistage du diabète de type 2 , c'est-à-dire l'épreuve d'hyperglycémie provoquée par voie orale (HGPO) de 2 heures. Cette étude a pour objet de déterminer si la mise en œuvre d'un système de rappel permet d'accroître les taux de dépistage.

Méthodologie : À la suite de notre essai comparatif randomisé (ECR) antérieur, nous avons intégré un protocole de rappel postnatal (par la poste ou par téléphone) aux pratiques des soins courants dans deux des trois sites cliniques. Nous avons vérifié dans quelle mesure les tests postnatals étaient réalisés en consultant les bases de données de laboratoire des hôpitaux et la base de données provinciale de facturation des services médicaux. Le paramètre primaire mesuré était la proportion de patientes soumises à une épreuve d'HGPO au cours des 6 mois ayant suivi l'accouchement.

Résultats : Les femmes ayant reçu des soins dans un établissement où était utilisé un système de rappel étaient plus nombreuses à subir une épreuve d'HGPO au cours des 6 mois suivant leur accouchement ( $28 \%$ ) que celles n’ayant pas bénéficié de ce système (14 \%). Pour les deux groupes ayant reçu un rappel, le taux d'HGPO (28\%) était inférieur à celui obtenu dans le cadre de notre ECR (60\%).

Conclusion : Le système de rappel postnatal a permis de doubler les taux de dépistage par l'épreuve d'HGPO recommandée, ceux-ci demeurant néanmoins faibles.

Mots-clés : diabète gestationnel, période postnatale, dépistage, système de rappel, prévention du diabète sucré de type 2

\section{Introduction}

Le diabète sucré gestationnel (DSG), défini comme étant une hyperglycémie au début de la grossesse ou reconnue pour la première fois au cours de la grossesse, touche environ $3 \%$ à $4 \%$ des femmes non autochtones et jusqu'à $18 \%$ des femmes autochtones au Canada ${ }^{1,2}$. Le DSG et l'intolérance au glucose (IG) pendant la grossesse sont associés à des résultats obstétricaux défavorables, mais le problème de santé publique le plus important pourrait être le risque 7 fois plus élevé que courent ces femmes de développer un diabète sucré de type 2 (DST2) par rapport à celles dont la tolérance au glucose est normale pendant la grossesse $\mathrm{e}^{3-6}$. L'Association canadienne du diabète (ACD) recommande de soumettre, entre 6 semaines et 6 mois après l'accouchement, les femmes ayant présenté un DSG à une épreuve d'hyperglycémie provoquée par voie orale (HGPO) de 2 heures? Les recommandations de la conférence internationale sur le DSG sont de procéder, 6 semaines après l'accouchement, à un test de dépistage du diabète sucré sous la forme d'une épreuve d'HGPO avec une charge de 75 grammes de glucose (HGPO $75 \mathrm{~g}$ ) de 2 heures ${ }^{7}$, cette épreuve devant être répétée un an après l'accouchement, puis au moins tous les 3 ans $^{8}$. Le test de glycémie à jeun utilisé seul ne décèle pas environ $40 \%$ des cas de diabète et ne permet pas de repérer les cas d'IG ${ }^{9}$. Or de $13 \%$ à $32 \%$ des femmes atteintes de DSG soumises à un test de dépistage entre 6 semaines et 3 mois après l'accouchement présentent une IG pouvant persister ou évoluer par la suite vers le DST2 ${ }^{10,11}$. La période postnatale s'avère donc un moment privilégié pour recenser les femmes courant un risque élevé d'être atteintes de diabète et, par le fait même, pour prendre des mesures de prévention et intervenir de façon précoce.

Bien que l'importance du dépistage postnatal au moyen de l'épreuve d'HGPO soit établie, les taux de dépistage demeurent malheureusement faibles dans le cadre de la pratique clinique courante ${ }^{12-14}$. Parmi les obstacles connus à la réalisation des tests de dépistage postnatals recommandés, on compte une mauvaise communication entre l'obstétricien et le fournisseur de soins primaires, la perplexité des fournisseurs devant les recommandations

\footnotetext{
Rattachement

1. Faculté de médecine, Université d'Ottawa, Ottawa (Ontario), Canada

2. Institut de recherche en services de santé, Toronto (Ontario), Canada

3. Faculté de médecine, Université de Toronto, Toronto (Ontario), Canada

4. Faculté de médecine, Centre Sunnybrook des sciences de la santé, Toronto (Ontario), Canada

5. Faculté de médecine, L'Hôpital d'Ottawa et Université d'Ottawa, Ottawa (Ontario), Canada

6. Obstétrique, Gynécologie et Soins aux nouveau-nés, L'Hôpital d'Ottawa et Université d'Ottawa, Ottawa (Ontario), Canada

7. Institut de recherche en santé d'Ottawa, Programme d'épidémiologie clinique, L'Hôpital d'Ottawa et l'Université d'Ottawa, Ottawa (Ontario), Canada

Correspondance : $\mathrm{D}^{\mathrm{re}}$ Erin Keely, Endocrinologie et métabolisme, L’Hôpital d'Ottawa, Campus Riverside, $4^{\mathrm{e}}$ étage, 1967, promenade Riverside, Ottawa (Ontario), Canada K1H 7W9; tél. : 613-738-8400 poste 81946; téléc. : 613-737-8396; courriel : ekeely@ottawahospital.on.ca
} 
en matière de dépistage, l'ignorance des patientes quant au risque qu'elles courent en l'absence de dépistage et leur omission de se présenter aux rendez-vous de dépistage en raison d'un conflit d'horaire ${ }^{15}$. Notre groupe a déjà montré que la plupart des Canadiennes de la région d'Ottawa, en Ontario, n'étaient pas soumises aux tests de dépistage postnatals recommandés ${ }^{12}$. Nous avons effectué un essai comparatif randomisé (ECR), à L'Hôpital d'Ottawa, dans le cadre duquel la femme, son médecin de famille, aucun des deux ou les deux recevaient par la poste, environ trois mois après l'accouchement, un rappel pour une épreuve d'HGPO. Lorsque la femme, le médecin ou les deux avaient reçu le rappel, les taux de dépistage étaient quatre fois plus élevés, passant de $14 \%$ (sans rappel) à environ $60 \%$. Environ $30 \%$ des femmes ayant subi l'épreuve d'HGPO avaient obtenu un résultat anormal ${ }^{16}$.

Nous nous sommes appuyés sur les résultats de cet ECR pour mettre en œuvre un système de rappel et l'intégrer aux pratiques courantes dans deux des trois sites cliniques de dépistage du DSG dans la région d'Ottawa. En raison de problèmes de logistique, la mise en place du système de rappel a été impossible dans le troisième site. Notre étude visait à déterminer si l'implantation de ce système de rappel dans les deux sites permettait de changer les taux de dépistage au moyen de l'épreuve d'HGPO de 2 heures recommandée par l'ACD. Nous avons posé l'hypothèse que le taux de dépistage postnatal par l'HGPO chez les femmes ayant reçu des soins aux sites où le système de rappel avait été implanté serait plus élevé que chez les autres femmes. Les analyses ont été fondées sur un modèle d'analyse en intention de traiter.

\section{Méthodologie}

Cette étude a été approuvée par le Conseil d'éthique en recherches de L'Hôpital d'Ottawa (L'HO) et le Comité d'éthique de la recherche de l'Hôpital Queensway Carleton (HQC). L'Hôpital d'Ottawa est un centre universitaire de soins tertiaires situé à Ottawa (Ontario, Canada) desservant environ 900000 habitants. Il offre des services obstétricaux sur deux de ses campus, soit le campus Civic (ouest de la ville) et le campus Général (est de la ville), avec environ 3500 accouchements pratiqués annuellement sur chacun d'eux. L'Hôpital Queensway Carleton (HQC), qui est un hôpital communautaire situé à la limite occidentale de la ville, dessert une population de plus de 400000 habitants et on y pratique 2800 accouchements par année. La plupart des femmes de la région sont orientées vers l'un de ces trois sites pour la prise en charge de leur DSG. Tous les soins primaires sont prodigués par des médecins de famille.

Nous avons recensé toutes les participantes aux séances d'information sur le DSG de l'un de ces trois sites entre le $1^{\text {er }}$ juillet 2007 et le 30 juin 2008. Elles avaient reçu un diagnostic de DSG après avoir passé soit une épreuve de charge en glucose de $50 \mathrm{~g}$, soit une épreuve d'HGPO avec une charge de $75 \mathrm{~g}$ de glucose (HGPO 75 g), conformément aux critères des Lignes directrices de pratique clinique de l'ACD ${ }^{7}$. Le diagnostic de DSG était posé 1) si la concentration de glucose plasmatique obtenue était supérieure ou égale à 10,3 mmol/l pour l'épreuve de charge en glucose de 50 g ou 2) si l'épreuve d'HGPO $75 \mathrm{~g}$ avait donné deux des trois résultats suivants : concentration de glucose plasmatique à jeun supérieure ou égale à 5,3 $\mathrm{mmol} / \mathrm{l}$, concentration de glucose plasmatique après 60 minutes supérieure ou égale à 10,6 mmol/l ou concentration de glucose plasmatique après 120 minutes supérieure ou égale à $8,9 \mathrm{mmol} / \mathrm{l}^{7}$. Les séances d'information sur le DSG présentent les risques associés au DSG, dont l'apparition du diabète après l'accouchement, et offrent des conseils nutritionnels personnalisés et des conseils sur la surveillance de la glycémie à la maison. Les patientes reviennent ensuite sur le site pour le traitement de leur DSG. Sur les sites A (L’HO, campus Général) et B (HQC), ceux utilisant le système de rappel, les femmes sont examinées par un endocrinologue/interniste chargé de leur prodiguer le traitement lié au DSG, et elles sont suivies par leur fournisseur habituel de soins obstétricaux pour leur grossesse, alors que sur le site n'utilisant pas le système de rappel (L'HO, campus Civic), les femmes sont examinées par un obstétricien spécialisé dans les grossesses à risque (GARE) et elles sont orientées vers le site pour y recevoir des soins obstétricaux si nécessaire. De plus, les patientes consultent un interniste si elles ont besoin d'aide pour le traitement par l'insuline. Les protocoles pour la mise en route d'une insulinothérapie sont similaires sur tous les sites et respectent les recommandations de l'ACD.

Tous les frais liés aux visites chez le médecin, aux soins médicaux et aux tests diagnostiques sont couverts par l'Assurancesanté de l'Ontario, qui est un régime provincial universel sans franchise. Les patientes non couvertes par l'Assurancesanté de l'Ontario ont été exclues de l'étude, car il était impossible de vérifier la réalisation des tests postnatals.

Environ trois mois après l'accouchement, les patientes du site A ont reçu par la poste un rappel soulignant l'importance des tests de dépistage du diabète et une réquisition pour une épreuve d'HGPO dans un laboratoire hors milieu hospitalier. Quant aux patientes du site $B$, elles ont reçu un appel téléphonique, une lettre accompagnée d'une demande d'analyse en laboratoire, ou les deux. Les patientes du site sans système de rappel n’ont reçu aucun rappel. Aucun site n'offrait de consultations de suivi systématique du DSG après l'accouchement.

Nous avons relevé les caractéristiques de base et les résultats obstétricaux des patientes en consultant leur dossier médical. Nous avons évalué leur statut socioéconomique à l'aide du quintile de revenu de leur quartier, selon le code postal de leur domicile. Aucune donnée sur l'ethnicité ou le niveau de scolarité n'était disponible. Pour recenser les tests de dépistage du diabète chez les participantes à l'étude, nous avons consulté le système de dossiers électroniques des hôpitaux, pour déterminer si les participantes avaient subi un test de dépistage du diabète postnatal au laboratoire de l'hôpital, ainsi que les dossiers de facturation des laboratoires communautaires. Nous avons relié chaque participante à la base de données provinciale de facturation des services médicaux au moyen de son numéro d'assurancemaladie unique, en utilisant les données de l'Institut de recherche en services de santé (IRSS). Comme le système de soins de santé de l'Ontario est un système universel à payeur unique, la base de données 
renferme les données sur toutes les analyses en laboratoire effectuées hors milieu hospitalier, y compris les types d'analyses et la date à laquelle elles ont été réalisées. (En revanche, les résultats des analyses en laboratoire ne peuvent pas être consultés à partir des bases.)

Le paramètre primaire visé était la proportion de patientes ayant subi, dans les six mois ayant suivi l'accouchement, une épreuve d'HGPO à des fins de dépistage du DST2. Deux autres paramètres ont été pris en compte également : 1) un test alternatif ayant pu être utilisé pour dépister le DST2 dans les six mois suivant l'accouchement (glycémie aléatoire, glycémie à jeun ou mesure de l'hémoglobine glycosylée [HbA1c]); 2) tout test ayant pu être utilisé pour dépister le DST2 dans les 6 mois suivant l'accouchement (HGPO, glycémie aléatoire, glycémie à jeun, glycémie plasmatique à jeun [GPJ] ou dosage de l'HbA1c).

Nous avons eu recours aux tests du chi carré et à l'analyse de la variance (ANOVA) pour comparer les paramètres correspondant aux données personnelles, cliniques et métaboliques de base entre les participantes à l'étude et les patientes exclues de l'étude, ainsi que pour comparer les trois sites. Pour comparer les taux de dépistage liés aux trois sites, nous avons effectué un test du chi carré pour chacun des résultats en fonction du site de l'accouchement. Nous avons effectué des analyses de régression logistique pour tenir compte des caractéristiques de base suivantes : âge maternel à l'accouchement, statut socio-économique, antécédents de DSG, indice de masse corporelle (IMC) avant la grossesse (normal, surpoids ou obésité), traitement du DSG (diète seulement ou insuline) et antécédents familiaux de DST2. Nous avons relevé les moyennes non ajustées et les écarts-types associés pour les variables continues, et la proportion de participantes en fonction de chacune des variables d'intérêt (nombre et pourcentage) pour les variables nominales. Les différences ont été jugées significatives si $p \leq 0,05$.

\section{Résultats}

Nous avons recensé au total 349 participantes aux séances d'information des trois sites. Parmi elles, 60 ne répondaient pas au critère de DSG et 27 n'étaient pas couvertes par l'Assurance-maladie de l'Ontario (principalement parce qu'elles étaient résidentes de la province voisine, c.-à-d. le Québec), ce qui a réduit l'effectif pour notre analyse à 262. Le taux de tabagisme était considérablement plus élevé chez les 27 femmes exclues que chez les participantes à l'étude $(p=0,002)$. De plus, le poids à la naissance des bébés des femmes exclues était plus faible ( $p=0,028$ ), mais cette différence n'était plus significative après la prise en compte de l'âge gestationnel à l'accouchement ( $p=0,316)$. Nous n'avons constaté aucune autre différence significative entre les femmes exclues et les participantes à l'étude (tableau 1).

La plupart des femmes ayant assisté aux séances d'information $(96,6 \%$; 253/262) sont revenues sur le site pour le traitement de leur DSG. Parmi les participantes des sites utilisant le système de rappel, le taux a été de $92,2 \%(83 / 90)$ pour le site $\mathrm{A}$ et $100 \%(55 / 55)$ pour le site B, et toutes ont été suivies par un interniste/endocrinologue pour leur traitement. Parmi les participantes du site n'utilisant pas le système de rappel, 98,3 \% (115/117) sont revenues sur le site pour leur traitement, et toutes sauf trois avaient consulté l'obstétricien spécialisé dans les grossesses à risque élevé pour le traitement de leur DSG. Parmi ces participantes, 17,9 \% (21/117) avaient aussi été examinées par un interniste/ endocrinologue.

Nous avons pu avoir accès au dossier des naissances de l'hôpital dans le cas de $91,6 \%$ des participantes (240/262). Aucune différence entre les sites n'a été observée pour les caractéristiques suivantes : poids à la naissance, proportion de nourrissons pesant plus de $4000 \mathrm{~g}$ à la naissance, proportion de césariennes proportion de grossesses multiples, proportion de primigestes ou proportion de femmes ayant présenté une prééclampsie (tableau 1). Par contre, les femmes du site n’utilisant pas le système de rappel avaient accouché plus tôt que celles fréquentant le site B (38 semaines contre 39 semaines, $p=0,010)$.

Un rappel par la poste accompagné d'une réquisition d'analyse en laboratoire a été envoyé à 96,7 \% des participantes du site A (3 non envoyés), et $11,5 \%(10 / 87)$ de ces envois ont été retournés en raison d'un changement d'adresse. Pour le site B, 76,3\% (42/55) ont reçu une lettre de rappel par la poste, $14,5 \%(8 / 55)$, un appel téléphonique d'un bénévole et 7,3\% (4/55), la lettre et l'appel; une participante n’a pu être jointe par téléphone et aucune lettre ne lui a été envoyée. Aucune des participantes du site sans système de rappel n'avait reçu de rappel par téléphone ou par la poste.

Dans l'analyse en intention de traiter, qui porte sur toutes les participantes, 21,7 \% $(57 / 262)$ des femmes avaient subi une épreuve d'HGPO pour le dépistage postnatal du diabète dans les 6 mois suivant l'accouchement : 23,3\% (21/90) du site A, $36,4 \%(20 / 55)$ du site B et $13,7 \%(16 / 117)$ du site sans système de rappel (tableau 2, $p=0,01)$. Si l'on combine les résultats des deux sites avec système de rappel, la proportion est de $28 \%$ (41/145), soit une plus plus forte proportion que celle liée au site sans système de rappel (chi carré $\left[\chi^{2}\right]=7,274 ; p=0,01$; degré de liberté $[\mathrm{nu}]=1)$. Dans les analyses de régression logique, un nombre significativement plus élevé de femmes du site $B$ ont subi une épreuve d'HGPO (rapport de cotes ajusté $[\mathrm{RC}]=3,10 ; p=0,03)$ si on compare avec celles du site sans système de rappel, le site A ne différant pas des autres relativement à la réalisation de l'épreuve d'HGPO (tableau 3). Quant passage d'un test quelconque de glycémie (glycémie aléatoire/ à jeun, dosage de l'HbA1c ou HGPO) au cours des 6 mois suivant l'accouchement, 41,6 \% (109/262) des femmes ont passé un ou plusieurs de ces tests. Pour les 57 femmes ayant subi une épreuve d'HGPO, cette dernière avait été consignée dans la base de données de facturation des laboratoires communautaires dans $81 \%$ des cas $(n=46)$. Aucune différence statistiquement significative n’a été observée entre les sites concernant la proportion 
TABLEAU 1

Caractéristiques personnelles, cliniques et métaboliques de base des participantes à l'étude

\begin{tabular}{|c|c|c|c|c|c|}
\hline & $\begin{array}{c}\text { Groupe } \\
\text { sans rappel } \\
(\mathrm{N}=117)\end{array}$ & $\begin{array}{l}\text { Groupe } A^{a} \\
\text { avec rappel } \\
(\mathrm{N}=90)\end{array}$ & $\begin{array}{l}\text { Groupe } B^{a} \\
\text { avec rappel } \\
(\mathrm{N}=55)\end{array}$ & $\begin{array}{l}\text { Exclues } \\
(N=27)\end{array}$ & $p$ \\
\hline \multicolumn{6}{|l|}{ Caractéristiques de base } \\
\hline Âge maternel à l’accouchement (années) & $34,0 \pm 5,4$ & $33,5 \pm 5,0$ & $33,3 \pm 4,0$ & $33,7 \pm 5,4$ & 0,659 \\
\hline $\begin{array}{l}\text { Âge gestationnel au diagnostic de DSG } \\
\text { (semaines) }\end{array}$ & $26,9 \pm 4,0$ & $27,8 \pm 2,8$ & $27,8 \pm 2,7$ & $26,6 \pm 3,4$ & 0,106 \\
\hline IMC avant la grossesse $\left(\mathrm{kg} / \mathrm{m}^{2}\right)$ & $27,6 \pm 6,8$ & $27,6 \pm 6,0$ & $25,4 \pm 5,3^{b}$ & $28,8 \pm 7,0$ & 0,039 \\
\hline \multicolumn{6}{|l|}{ Catégorie d'IMC (n, \%) } \\
\hline Inconnu & $0(0,0)$ & $27(30,0)$ & $1(1,8)$ & & \\
\hline Normal & $53(45,3)$ & $23(25,6)$ & $28(50,9)$ & & \\
\hline Surpoids & $28(23,9)$ & $23(25,6)$ & $13(23,6)$ & & \\
\hline Obésité & $36(30,8)$ & $17(18,9)$ & $13(23,6)$ & & \\
\hline Antécédents de DSG & $30(26,3)$ & $20(22,5)$ & $6(11,1)$ & $5(18,5)$ & 0,082 \\
\hline Primigeste & $35(29,9)$ & $27(30,0)$ & $16(29,1)$ & $5(18,5)$ & 0,992 \\
\hline Tabagisme pendant la grossesse & $8(6,8)$ & $2(2,6)$ & $5(9,1)$ & $6(22,2)$ & 0,258 \\
\hline Glycémie à l'HGPO 50 g (mmol/l) & $11,1 \pm 2,2$ & $11,8 \pm 1,8$ & $11,2 \pm 1,7$ & $11,5 \pm 1,9$ & 0,202 \\
\hline $\begin{array}{l}\text { HGPO : glycémie } 0 \mathrm{~min} \text { après l'ingestion de } \\
75 \mathrm{~g} \text { de glucose }(\mathrm{mmol} / \mathrm{l})\end{array}$ & $5,4 \pm 0,8$ & $5,5 \pm 0,7$ & $5,2 \pm 0,7$ & $5,4 \pm 0,7$ & 0,35 \\
\hline $\begin{array}{l}\text { HGPO : glycémie } 60 \mathrm{~min} \text { après l'ingestion de } \\
75 \mathrm{~g} \text { de glucose }(\mathrm{mmol} / \mathrm{l})\end{array}$ & $11,8 \pm 1,3$ & $11,6 \pm 1,4$ & $11,7 \pm 0,9$ & $11,5 \pm 1,1$ & 0,803 \\
\hline $\begin{array}{l}\text { HGPO : glycémie } 120 \text { min après l'ingestion de } \\
75 \text { g de glucose (mmol/l) }\end{array}$ & $9,4 \pm 1,7$ & $9,6 \pm 1,8$ & $9,6 \pm 1,4$ & $9,3 \pm 1,3$ & 0,835 \\
\hline \multicolumn{6}{|l|}{ Traitement du DSG } \\
\hline DSG traité à l'insuline & $45(38,5)$ & $25(27,8)$ & $14(25,5)$ & $8(29,6)$ & 0,131 \\
\hline \multicolumn{6}{|l|}{ Issue de la grossesse } \\
\hline Âge gestationnel à l'accouchement (semaines) & $38,0 \pm 2,2$ & $38,7 \pm 2,5$ & $39,0 \pm 1,5^{b}$ & $37,3 \pm 3,3$ & 0,01 \\
\hline Poids du bébé à la naissance (grammes) & $3280,4 \pm 682,6$ & $3408,6 \pm 690,0$ & $3440,7 \pm 513,8$ & $3058,3 \pm 670,0$ & 0,233 \\
\hline Grossesse multiple & $6(5,2)$ & $2(2,8)$ & $0(0,0)$ & $2(7,7)$ & 0,196 \\
\hline Hypertension gravidique & $12(10,4)$ & $3(4,2)$ & $5(9,1)$ & $5(19,2)$ & 0,655 \\
\hline Prééclampsie & $7(6,1)$ & $4(5,6)$ & $2(3,6)$ & $0(0,0)$ & 0,655 \\
\hline Césarienne & $50(43,9)$ & $27(37,5)$ & $20(36,4)$ & $12(46,2)$ & 0,552 \\
\hline Antécédents familiaux de DST2 & $59(50,4)$ & $51(56,6)$ & $27(49,1)$ & $13(48,1)$ & 0,527 \\
\hline \multicolumn{6}{|l|}{ Quintile de revenu (selon le code postal) } \\
\hline Données manquantes & $2(1,7)$ & $1(1,1)$ & $0(0,0)$ & s.o. & $0,001^{c}$ \\
\hline 1 (inférieur) & $32(27,4)$ & $32(35,6)$ & $3(5,5)$ & S.o. & \\
\hline 2 & $19(16,2)$ & $12(13,3)$ & $13(23,6)$ & S.o. & \\
\hline 3 & $27(23,1)$ & $17(18,9)$ & $13(23,6)$ & S.o. & \\
\hline 4 & $19(16,2)$ & $22(24,4)$ & $10(18,2)$ & s.o. & \\
\hline 5 (supérieur) & $18(15,4)$ & $6(6,7)$ & $16(29,1)$ & s.o. & \\
\hline
\end{tabular}

Abréviations : DSG, diabète sucré gestationnel; DST2, diabète sucré de type 2; HGPO, épreuve d'hyperglycémie provoquée par voie orale; IMC, indice de masse corporelle; N, taille de l'échantillon; $p$, signification statistique.

Notes : s.o. = données non disponibles en raison de l'absence du numéro d'assurance-maladie provincial permettant de lier les données.

Les données représentent une moyenne ( \pm écart-type) ou un nombre (\%).

${ }^{a}$ Les groupes A et B avec rappel ont été comparés au groupe sans rappel au moyen de tests du chi carré et de l'analyse de la variance (ANOVA).

${ }^{\mathrm{b}}$ Significatif par rapport au groupe sans rappel.

' Différence significative, d'après le quintile de revenu, entre le groupe B avec rappel, d'une part, et le groupe A avec rappel et le groupe sans rappel, d'autre part. 
de femmes ayant subi un test de glycémie aléatoire/à jeun, un dosage de l'HbA1c ou tout autre test de dépistage du diabète.

Les facteurs pouvant influer sur les taux de dépistage ont été intégrés à l'analyse de régression logistique (tableau 3 ). Les femmes dont le traitement consistait en une diète seulement (par opposition à celle suivant un traitement par insuline) étaient moins susceptibles de subir une épreuve d'HGPO (RC ajusté $=0,38$; IC $=0,18-$ $0,80 ; p=0,01)$. Aucun autre facteur n'a été considéré comme prédicteur significatif de la réalisation de l'épreuve d'HGPO. Paradoxalement, les femmes ayant un poids normal ou un surpoids (par opposition aux femmes obèses) étaient plus nombreuses à subir l'un ou l'autre des tests de glycémie au cours de la période postnatale ( $\mathrm{RC}$ ajusté $=2,40 ; \mathrm{IC}=1,16-5,01$ et $\mathrm{RC}$ ajusté $=3,10$; IC $=1,42-6,77$, respectivement; $p=0,03)$. Les antécédents familiaux de DST2 et les antécédents personnels de DSG n'ont pas influencé de façon significative le fait que la participante subisse un test de dépistage postnatal du diabète.

\section{Analyse}

Nous avons observé que les femmes ayant des antécédents de DSG qui ont reçu des soins dans des sites utilisant un système de rappel à des fins de dépistage postnatal du diabète étaient plus susceptibles de subir le test recommandé (l'épreuve d'HGPO). Pour l'ensemble des participantes ayant reçu un rappel, le pourcentage de celles ayant passé une épreuve d'HGPO était inférieur à celui obtenu dans le cadre de notre ECR, soit $28 \%$ contre $60 \%$, ce qui était à prévoir dans le cadre d'une étude d'efficacité comparative. Aucune amélioration de nos taux de dépistage sans rappel n'a été constatée $(13,7 \%$ des femmes du site sans système de rappel ont subi l'épreuve d'HGPO, taux similaire à celui des femmes du groupe sans intervention dans l'ECR, soit 14,3\%). Les taux de dépistage au moyen de l'épreuve d'HGPO étaient inférieurs sur le site A, mais il faut noter que $14,4 \%$ des participantes de ce site n'avaient pas reçu effectivement le rappel par courriel ou l'appel téléphonique. Selon le principe d'analyse en intention de traiter, ces femmes doivent cependant toujours demeurer incluses dans les analyses, et les participantes de ce groupe demeuraient malgré tout plus nombreuses à subir l'épreuve d'HGPO que celles sans rappel.

Nos résultats révèlent que le nombre de participantes du site sans système de rappel ayant subi une épreuve d'HGPO était significativement inférieur à celui des femmes des sites avec système de rappel, mais aucune différence n'a été observée quant à la proportion de femmes ayant passé d'autres tests de glycémie (glycémie aléatoire, glucose plasmatique à jeun [GPJ] ou dosage de l'HbA1c). De manière générale, une réticence à se soumettre à une épreuve d'HGPO a été observée au sein de la population de l'Ontario ${ }^{17}$, quoiqu'il s'agisse du meilleur test de dépistage du diabète, les autres types de tests pouvant entraîner des

TABLEAU 2

Proportion de sujets dans chaque groupe ayant subi un test de glycémie à des fins de dépistage au cours des 6 mois suivant l'accouchement.

\begin{tabular}{|c|c|c|c|c|}
\hline \multirow[b]{2}{*}{ HGPO } & \multirow{2}{*}{$\begin{array}{c}\text { Groupe } \\
\text { sans rappel } \\
(\mathbf{N}=117) \\
16(13,7 \%)\end{array}$} & $\begin{array}{c}\text { Groupe } A \\
\text { avec rappel } \\
(\mathbf{N}=90)\end{array}$ & \multicolumn{2}{|c|}{$\begin{array}{c}\text { Groupe B } \\
\text { avec rappel } \\
(\mathrm{N}=55)\end{array}$} \\
\hline & & $21 \quad(23,3 \%)$ & 20 & $(36,4 \%)^{a}$ \\
\hline $\begin{array}{l}\text { Mesure aléatoire/à jeun de la } \\
\text { glycémie }\end{array}$ & $31(26,5 \%)$ & $23(25,6 \%)$ & 12 & $(21,8 \%)$ \\
\hline Dosage de l'HbA1c & $16(13,7 \%)$ & $12 \quad(13,3 \%)$ & 9 & $(16,4 \%)$ \\
\hline Tout test de glycémie & $44(37,6 \%)$ & $38 \quad(42,2 \%)$ & 27 & $(49,1 \%)$ \\
\hline
\end{tabular}

Abréviations : HbA1c, hémoglobine glycosylée; HGPO, épreuve d'hyperglycémie provoquée par voie orale;

$\mathrm{N}$, taille de l'échantillon; $p$, signification statistique.

${ }^{\text {a }} p=0,01$, groupe $\mathrm{B}$ avec rappel par rapport au groupe sans rappel.

résultats faussement négatifs. Selon une étude de cohorte à grande échelle portant sur des femmes ayant des antécédents de DSG et ayant subi à la fois l'épreuve d'HGPO et le test de GPJ, le test de GPJ réalisé seul n'aurait pas permis de diagnostiquer le diabète de $38 \%$ des femmes présentant un état prédiabétique et de $75 \%$ de celles répondant aux critères du diabète de type $2^{18}$. De même, une étude canadienne a révélé que lorsque les résultats du test de GPJ étaient utilisés seuls, $54 \%$ des femmes atteintes de diabète étaient considérés comme ayant une glycémie normale ${ }^{9}$. Dans notre étude, seulement la moitié des femmes ayant fait l'objet d'un test de dépistage avaient subi le test recommandé (HGPO dans $21,7 \%$ des cas, tout autre test dans 41,6 \% des cas), ce qui laisse penser que les lignes directrices de l'ACD ne sont pas suivies et, par le fait même, que les possibilités d'intervention précoce sont limitées. Avec des niveaux d'intolérance au glucose semblables à la base, les femmes ayant des antécédents de DSG courent plus de risque d'être atteintes du DST2 dans les 3 années qui suivent que celles sans antécédent de DSG $(38,4 \% \text { contre } 25,7 \%)^{19}$. Bon nombre d'essais cliniques ont démontré que l'apparition du DST2 peut être retardée, sinon prévenue, chez les patientes à haut risque lorsque celles-ci changent leurs habitudes de vie et se tournent vers la pharmacothérapie ${ }^{19-22}$. En fait, cette dernière serait davantage bénéfique aux femmes ayant des antécédents de DSG ${ }^{19}$. Il convient donc de continuer à sensibiliser les fournisseurs de soins de santé et les patientes, et d'offrir des services novateurs, afin d'accroître l'utilisation de l'épreuve d'HGPO.

Plusieurs facteurs peuvent influer sur le passage de tests de dépistage postnatals. La prestation de service diffère entre le site sans système de rappel et les sites avec système de rappel. Dans les sites avec système de rappel, toutes les patientes avaient été examinées par un interniste/endocrinologue, alors que dans le site sans système de rappel, elles avaient consulté un obstétricien spécialisé dans les grossesses à risque élevé, et un interniste seulement pour faire ajuster leurs doses d'insuline. Cependant, étant donné que le taux de dépistage était le même que celui obtenu dans le groupe 
TABLEAU 3

Régression logistique ajustée pour prévoir le dosage de la glycémie à des fins de dépistage après l'accouchement chez les femmes ayant des antécédents de DSG.

\begin{tabular}{|c|c|c|c|c|}
\hline Résultat & Effet & RC ajusté & Intervalle de confiance [IC] à 95 \% & $p$ \\
\hline \multicolumn{5}{|l|}{ HGPO } \\
\hline \multicolumn{2}{|r|}{ Site } & & & 0,029 \\
\hline \multirow{2}{*}{\multicolumn{2}{|c|}{$\begin{array}{l}\text { Groupe A avec rappel vs } \\
\text { groupe sans rappel } \\
\text { Groupe B avec rappel vs } \\
\text { groupe sans rappel }\end{array}$}} & 1,57 & $0,66-3,70$ & \\
\hline & & 3,10 & $1,35-7,14$ & \\
\hline \multicolumn{2}{|r|}{ Âge } & & & 0,262 \\
\hline \multicolumn{2}{|r|}{$<30$ ans vs $\geq 40$ ans } & 2,51 & $0,58-10,83$ & \\
\hline \multicolumn{2}{|r|}{$30-39$ ans vs $\geq 40$ ans } & 3,06 & $0,79-11,84$ & \\
\hline \multicolumn{2}{|r|}{ Antécédents de DSG } & 0,49 & $0,20-1,23$ & 0,131 \\
\hline \multicolumn{2}{|r|}{ IMC } & & & 0,134 \\
\hline \multicolumn{2}{|r|}{ Normal vs obésité } & 2,42 & $0,92-6,36$ & \\
\hline \multicolumn{2}{|r|}{ Surpoids vs obésité } & 3,30 & $1,20-9,06$ & \\
\hline \multicolumn{5}{|c|}{ Traitement du DSG } \\
\hline \multicolumn{2}{|r|}{ Diète vs insuline } & 0,38 & $0,18-0,80$ & 0,012 \\
\hline \multicolumn{2}{|r|}{ Antécédents familiaux de DST2 } & 1,07 & $0,55-2,05$ & 0,845 \\
\hline \multicolumn{2}{|r|}{ Quintiles du SSE } & & & 0,635 \\
\hline \multicolumn{2}{|r|}{1 (inférieur) vs 5 (supérieur) } & 0,89 & $0,29-2,63$ & \\
\hline \multicolumn{2}{|r|}{2 vs 5} & 0,96 & $0,33-2,84$ & \\
\hline \multicolumn{2}{|r|}{3 vs 5} & 0,85 & $0,29-2,47$ & \\
\hline \multicolumn{2}{|r|}{4 vs 5} & 1,68 & $0,59-4,77$ & \\
\hline \multicolumn{5}{|c|}{ Tout autre test } \\
\hline \multicolumn{2}{|r|}{ Site } & & & 0,734 \\
\hline \multicolumn{2}{|r|}{$\begin{array}{l}\text { Groupe A avec rappel vs } \\
\text { groupe sans rappel }\end{array}$} & 1,09 & $0,56-2,13$ & \\
\hline \multicolumn{2}{|r|}{$\begin{array}{l}\text { Groupe B avec rappel vs } \\
\text { groupe sans rappel }\end{array}$} & 1,33 & $0,65-2,71$ & \\
\hline \multicolumn{2}{|r|}{ Âge } & & & 0,595 \\
\hline \multicolumn{2}{|r|}{$<30$ ans vs $\geq 40$ ans } & 0,74 & $0,28-1,98$ & \\
\hline \multicolumn{2}{|r|}{$30-39$ ans vs $\geq 40$ ans } & 1,05 & $0,44-2,49$ & \\
\hline \multicolumn{2}{|r|}{ Antécédents de DSG } & 0,68 & $0,35-1,34$ & 0,264 \\
\hline \multicolumn{2}{|r|}{ IMC } & & & 0,032 \\
\hline & Normal vs obésité & 2,40 & $1,16-5,01$ & \\
\hline & Surpoids vs obésité & 3,10 & $1,42-6,77$ & \\
\hline & Traitement du DSG & & & \\
\hline & Diète vs insuline & 0,60 & $0,32-1,12$ & 0,107 \\
\hline & Antécédents familiaux de DST2 & 0,77 & $0,45-1,31$ & 0,328 \\
\hline & Quintiles du SSE & & & 0,195 \\
\hline & 1 (inférieur) vs 5 (supérieur) & 0,92 & $0,38-2,21$ & \\
\hline & 2 vs supérieur & 1,33 & $0,54-3,31$ & \\
\hline & 3 vs supérieur & 0,85 & $0,35-2,04$ & \\
\hline & 4 vs supérieur & 2,09 & $0,85-5,13$ & \\
\hline
\end{tabular}

Abréviations : DSG, diabète sucré gestationnel; DST2, diabète sucré de type 2; HGPO, épreuve d’hyperglycémie provoquée par voie orale; IC, intervalle de confiance; IMC, indice de masse corporelle; RC, rapport de cotes; SSE, statut socio-économique.

Notes : La catégorie figurant en premier sur chaque ligne représente le groupe de référence. 
sans intervention de notre ECR, et que le modèle de soins était le même (à part le système de rappel postnatal) dans tous les sites, il y a tout lieu de penser que la différence découle de la mise en œuvre du système de rappel. De plus, malgré les efforts déployés pour que les patientes reçoivent le rappel et soient disponibles pour le dépistage, quatre participantes n'avaient pas reçu le rappel et dix rappels envoyés par la poste avaient été retournés, ce qui signifie que 9,6 \% des femmes visées par le rappel avaient été perdues de vue. En outre, les participantes de l'ECR savaient qu'elles faisaient l'objet d'une étude de recherche, alors que les femmes de cette étude l'ignoraient.

Il semble que des consultations plus fréquentes auprès de fournisseurs de soins de santé permettent d'augmenter les taux de dépistage. En effet, on a constaté que les taux de dépistage étaient plus élevés lorsque les femmes consultaient des fournisseurs de soins de santé au cours de la période postnatale, soit un endocrinologue, soit un fournisseur prescrivant le test lors de la consultation de suivi systématique prévue 6 semaines après l'accouchement ${ }^{23,24}$. De même, dans le cadre d'une étude de cohorte portant sur plus de 14000 femmes atteintes du DSG, les consultations auprès d'un interniste ou d'un gynécologue obstétricien constituaient des prédicteurs indépendants et significatifs du dépistage postnatal dans l'année suivant l'accouchement ${ }^{18}$. Dans le cadre de notre pratique, aucun rendez-vous postnatal systématique n'est fixé avec l'interniste/ endocrinologue. Notre étude a été limitée par le fait que nous n'avions pas accès aux dossiers relatifs aux soins postnatals fournis par un obstétricien ou un fournisseur de soins primaires hors milieu hospitalier.

Bien qu'aucune autre étude directe ne compare des modèles de prestation de soins, le système par gestion de cas peut s'avérer efficace pour augmenter les taux de dépistage. Dans le cadre d'une étude prospective de cohorte, des femmes atteintes de DSG ont fait l'objet d'un suivi : elles ont reçu des réquisitions d'analyse en laboratoire à leur congé de l'hôpital et ont été appelées à la maison par un gestionnaire de cas pouvant réaliser le test de dépistage. Cette mesure a fait passer le taux de dépistage par HGPO à $41 \%{ }^{25}$. Au centre Kaiser Permanente dans le nord de la Californie, les taux de dépistage par l'épreuve d'HGPO sont passés de $16,6 \%$ à 71,5 \% lorsqu'un programme de soins géré par le personnel infirmier a été instauré $^{18}$.

Les femmes courant un risque plus élevé de développer le DST2 ne retournent pas forcément passer un test de dépistage. Dans notre étude, les femmes traitées à l'insuline étaient plus disposées à faire l'objet d'un dépistage postnatal que les femmes obèses, 28,8 \% seulement de ces dernières ayant subi un test quelconque de glucose. Dans le cadre d'une étude prospective de cohorte, des femmes atteintes du DSG ont fait l'objet d'un suivi; elles ont reçu une demande d'analyse en laboratoire à leur congé de l'hôpital, de même qu'un appel à la maison d'un gestionnaire de cas pouvant réaliser le test de dépistage ${ }^{25}$. Les femmes ne s'étant pas présentées pour un test de dépistage étaient plus nombreuses à avoir des antécédents de DSG, avaient une glycémie plus élevée au moment du diagnostic et étaient plus susceptibles d'avoir pris de l'insuline durant leur grossesse, ce qui donne à penser que les femmes souffrant d'une forme moins grave de DSG étaient plus disposées à faire l'objet d'un suivi. Des recherches plus poussées sur les perceptions du risque en regard du dépistage s'avèrent donc essentielles pour comprendre pourquoi les femmes courant un risque plus élevé d'être atteintes de diabète ne se soumettent pas à des tests de dépistage.

Notre étude comporte toutefois plusieurs limites. Le suivi des femmes conformément aux recommandations de l'ACD en matière de dépistage ne s'est déroulé que sur une période de 6 mois après l'accouchement. (Dans le cadre de notre ECR, nous avions inclus les tests réalisés sur une période d'un an.) Les résultats d'autres études varient : une étude a révélé que des femmes attendaient jusqu'à 428 jours après l'accouchement pour subir des tests ${ }^{14}$, alors qu'une autre étude a démontré que 94,3\% des femmes subissaient ces tests au cours des 12 semaines suivant l'accouchement ${ }^{25}$. Cependant, il se peut que la généralisabilité des résultats soit limitée par le fait que cette étude a été réalisée dans un seul centre urbain multiculturel.

En résumé, les rappels constituent un moyen efficace pour renforcer l'application des lignes directrices en matière de dépistage du diabète après l'accouchement. La plupart des femmes ne subissent cependant toujours pas de test de glycémie, encore moins l'épreuve d'HGPO recommandée. Les fournisseurs de soins de santé doivent donc envisager de mettre en place une méthode structurée visant à assurer le suivi postnatal des femmes ayant des antécédents de DSG. D’autres études devraient évaluer différents systèmes de rappel après l'accouchement et analyser les obstacles à leur mise en œuvre.

\section{Remerciements}

Nous tenons à remercier Sharon Rouatt, éducatrice spécialisée en diabétologie; Pauline Brazeau-Gravelle, diététiste clinique; Filamena DeSousa, éducatrice spécialisée en diabétologie; et Anjie Huang, statiscienne. Les instituts de recherche en santé du Canada (IRSC) ont accordé à Alison Shea une bourse de stagiaire de recherche. L'étude a été financée par le groupe de recherche Obstetrics Maternal \& Newborn Investigation (OMNI) et L'Hôpital d'Ottawa. Le Dr Baiju Shah a reçu un appui salarial des IRSC, de l'Association canadienne du diabète et du Banting and Best Diabetes Centre de l'Université de Toronto. Les auteurs ne présentent aucun conflit d’intérêt en lien avec cette étude.

\section{Références}

1. Dyck R, Klomp H, Tan LK, Turnell RW, Boctor MA. A comparison of rates, risk factors, and outcomes of gestational diabetes between aboriginal and non-aboriginal women in the Saskatoon health district. Diabetes Care. 2002;25:487-93.

2. Rodrigues S, Robinson E, Gray-Donald K. Prevalence of gestational diabetes mellitus among James Bay Cree women in northern Quebec. CMAJ. 1999;160:1293-7. 
3. Feig DS, Zinman B, Wang X, Hux JE. Risk of development of diabetes mellitus after diagnosis of gestational diabetes. CMAJ. 2008;179:229-34.

4. Kim C, Newton KM, Knopp RH. Gestational diabetes and the incidence of type 2 diabetes: a systematic review. Diabetes Care. 2002;25:1862-68.

5. Retnakaran R, Qi Y, Sermer M, Connelly PW, Hanley AJ, Zinman B. Glucose intolerance in pregnancy and future risk of pre-diabetes or diabetes. Diabetes Care. 2008;31:2026-31.

6. Bellamy L, Casas JP, Hingorani AD, Williams D. Type 2 diabetes mellitus after gestational diabetes: a systematic review and meta-analysis. Lancet. 2009;373:1773-9.

7. Comité d'experts des Lignes directrices de pratique clinique de l'Association canadienne du diabète. Lignes directrices de pratique clinique 2008 de l'Association canadienne du diabète pour la prévention et le traitement du diabète au Canada. Can J Diabetes. 2008: 32 (suppl. 2) ; S1 à S225.

8. Metzger BE, Buchanan TA, Coustan DR, de Leiva A, Dunger DB, Hadden DR, Hod M, Kitzmiller JL, Kjos SL, Oats JN, Pettitt DJ, Sacks DA, Zoupas C. Summary and recommendations of the Fifth International Workshop-Conference on Gestational Diabetes Mellitus. Diabetes Care. 2007;30 Suppl 2:S251-60.

9. Reinblatt SL, Morin L, Meltzer SJ. The importance of a postpartum $75 \mathrm{~g}$ oral glucose tolerance test in women with gestational diabetes. J Obstet Gynaecol Can. 2006;28:690-4

10. Ogonowski J, Miazgowski T. The prevalence of 6 weeks postpartum abnormal glucose tolerance in Caucasian women with gestational diabetes. Diabetes Res Clin Pract. 2009;84:239-44.

11. Retnakaran R, Qi Y, Sermer M, Connelly PW, Zinman B, Hanley AJ. Comparison of National Diabetes Data Group and American Diabetes Association diagnostic criteria for gestational diabetes in their identification of postpartum risk of glucose intolerance. Diabetes Res Clin Pract. 2009;85:40-6.

12. Clark HD, van Walraven C, Code C, Karovitch A, Keely E. Did publication of a clinical practice guideline recommendation to screen for type 2 diabetes in women with gestational diabetes change practice? Diabetes Care. 2003;26:265-8.

13. Dietz PM, Vesco KK, Callaghan WM, Bachman DJ, Bruce FC, Berg CJ, England LJ, Hornbrook MC. Postpartum screening for diabetes after a gestational diabetes mellitus-affected pregnancy. Obstet Gynecol. 2008;112:868-74.

14. Smirnakis KV, Chasan-Taber L, Wolf M, Markenson G, Ecker JL, Thadhani R. Postpartum diabetes screening in women with a history of gestational diabetes. Obstet Gynecol. 2005;106:1297-303.

15. Bentley-Lewis R, Levkoff S, Stuebe A, Seely EW. Gestational diabetes mellitus: postpartum opportunities for the diagnosis and prevention of type 2 diabetes mellitus. Nat Clin Pract Endocrinol Metab. 2008;4:552-8.

16. Clark HD, Graham ID, Karovitch A, Keely EJ. Do postal reminders increase postpartum screening of diabetes mellitus in women with gestational diabetes mellitus? A randomized controlled trial. Am J Obstet Gynecol. 2009;200:634.e1-7.

17. Wilson SE, Lipscombe LL, Rosella LC, Manuel DG. Trends in laboratory testing for diabetes in Ontario, Canada 1995-2005: a population-based study. BMC Health Serv Res. 2009;9:41.

18. Ferrara A, Peng T, Kim C. Trends in postpartum diabetes screening and subsequent diabetes and impaired fasting glucose among women with histories of gestational diabetes mellitus: a report from the Translating Research Into Action for Diabetes (TRIAD) Study. Diabetes Care. 2009;32:269-74.

19. Ratner RE, Christophi CA, Metzger BE, Dabelea D, Bennett PH, Pi-Sunyer X, Fowler S, Kahn SE; Diabetes Prevention Program Research Group. Prevention of diabetes in women with a history of gestational diabetes: effects of metformin and lifestyle interventions. J Clin Endocrinol Metab. 2008;93:4774-9.

20. Ratner RE. Prevention of type 2 diabetes in women with previous gestational diabetes. Diabetes Care. 2007;30:S242-5.

21. Xiang AH, Peters RK, Kjos SL, Marroquin A, Goico J, Ochoa C, Kawakubo M, Buchanan TA. Effect of pioglitazone on pancreatic beta-cell function and diabetes risk in Hispanic women with prior gestational diabetes. Diabetes. 2006;55:517-22.

22. Buchanan TA, Xiang AH, Peters RK, Kjos SL, Marroquin A, Goico J, Ochoa C, Tan S, Berkowitz K, Hodis HN, Azen SP. Preservation of pancreatic beta-cell function and prevention of type 2 diabetes by pharmacological treatment of insulin resistance in high-risk Hispanic women. Diabetes. 2002;51:2796-803.

23. Kim C, Tabaei BP, Burke R, McEwen LN, Lash RW, Johnson SL, Schwartz KL, Bernstein SJ, Herman WH. Missed opportunities for type 2 diabetes mellitus screening among women with a history of gestational diabetes mellitus. Am J Public Health. 2006;96:1643-8

24. Greenberg LR, Moore TR, Murphy H. Gestational diabetes mellitus: antenatal variables as predictors of postpartum glucose intolerance. Obstet Gynecol. 1995;86:97-101.

25. Hunt KJ, Conway DL. Who returns for postpartum glucose screening following gestational diabetes mellitus? Am J Obstet Gynecol. 2008;198:404.e1-6. 